\title{
La oximetría de pulso ayuda a detectar cardiopatías congénitas dependientes del ductus
}

Pulse oximetry helps detect ductus dependent congenital heart disease

Anne de-Wahl Granelli y col. BMJ 2009;338;a3037

\section{Objetivo}

Evaluar la oximetría de pulso $(\mathrm{SpO} 2)$ para el rastreo de cardiopatías congénitas.

\section{Diseño, lugar y pacientes}

Corte transversal y cohorte que comparó la detección de cardiopatías dependientes del flujo ductal (CCFD) en Götaland (Suecia) con la de otras regiones que no utilizan oximetría de pulso. Fue llevado a cabo entre 2004 y 2007 en maternidades y centros de referencia regionales e incluyó 39.821 recién nacidos $(\mathrm{RN})$.

Descripción del test y medición de los resultados principales Medición de la SpO2 pre y post-ductal al alta de la maternidad, considerándosela una prueba positiva ante una $\mathrm{SpO} 2$ menor a
$95 \%$ ó una diferencia entre ambas mayor a 3\%. El examen físico fue ciego a los resultados de la oximetría, excepto cuando esta era menor a $90 \%$. El test de referencia fueron la ecocardiografía y el seguimiento de todos los pacientes. Fueron evaluadas la sensibilidad y especificidad, los valores predictivos y los coeficientes de probabilidad para la pesquisa con $\mathrm{SpO}$, con examen físico (EF) aislado y con la combinación de ambos.

\section{Resultados Principales}

En West Götaland 29 RN asintomáticos al alta tenían una (CCFD). En 13 casos, la SpO2 fue menor a 90 y en los 16 restantes, el EF aislado detectó a diez (63\%). La pesquisa combinada de EF más SpO2 tuvo una sensibilidad de $83 \%$ (IC95\% 64 a 95) y tuvo un valor predictivo negativo cercano al $100 \%$. Ver tabla 1.

Tabla 1: características operativas de los métodos de rastreo cardiopatías congénitas dependientes de flujo ductal.

\begin{tabular}{|c|c|c|c|c|c|c|}
\hline & \multirow{2}{*}{$\begin{array}{l}\text { Sensibilidad } \\
\text { (IC95\%) }\end{array}$} & \multirow{2}{*}{$\begin{array}{l}\text { Especificidad } \\
\text { (IC95\%) }\end{array}$} & \multicolumn{2}{|c|}{ Valor predictivo (IC95\%) } & \multicolumn{2}{|c|}{ Coeficiente de probabilidad (IC95\%) } \\
\hline & & & Positivo & Negativo & Positivo (LR+) & Negativo (LR-) \\
\hline $\begin{array}{l}\text { Oximetría } \\
\text { de pulso }\end{array}$ & $\begin{array}{c}62 \% \\
(42 \text { a } 79)\end{array}$ & $\begin{array}{c}99,8 \% \\
(99,77 \text { a } 99,86)\end{array}$ & $\begin{array}{c}20,7 \\
(12,7 \text { a } 30,7)\end{array}$ & $\begin{array}{c}99,97 \\
(99,95 \text { a } 99,99)\end{array}$ & 345 & 0,38 \\
\hline $\begin{array}{c}\text { Oximetria de pulso más } \\
\text { examen físico }\end{array}$ & $\begin{array}{c}83 \% \\
(64 \text { a } 95)\end{array}$ & $\begin{array}{c}97,9 \% \\
(97,7 \text { a } 98)\end{array}$ & $\begin{array}{c}2,9 \\
(1,9 \text { a } 4,3)\end{array}$ & $\begin{array}{c}99,99 \\
(99,97 \text { a 100) }\end{array}$ & 39 & 0,17 \\
\hline
\end{tabular}

Conclusiones

La introducción del rastreo con $\mathrm{SpO}_{2}$ al alta aumentó la detección de CCFD en RN asintomáticos a $83 \%$. La probable prevención de morbilidad neurológica y una menor necesidad de cuidados intensivos pre-quirúrgicos sugieren que esta pesquisa podría ser costo-efectiva en el largo plazo.
Palabras claves: cardiopatía congénita, oximetría percutánea, pesquisa, recién nacido. Keywords: congenital heart disease, percutaneous oximetry, screening, newborn. Fuentes de financiamiento: Regional Science and Education Foundation y Gothenburg University.

Comentario

Uno a dos por mil RN presentan una CCFD potencialmente grave y cerca de $35 \%$ de esos pacientes son dados de alta de la maternidad sin diagnosticar, lo que compromete seriamente sus probabilidades de supervivencia. Como la combinación de un adecuado control prenatal con una evaluación cuidadosa luego del nacimiento mejora el pronóstico', la utilización de la SpO2 de última generación había sido sugerida como una herramienta útil en estos pacientes ${ }^{2}$. Este estudio tiene un excelente diseño $\left.{ }^{3}: 1\right)$ comparación independiente con una buena prueba de referencia como lo es la ecocardiografía; 2) la falta de enmascaramiento está éticamente justificada y subsanada a través de un estudio complementario de cohortes; 3 ) la muestra es adecuada y representativa de la población de interés.

Sus resultados son importantes, han sido expresados con adecuada precisión y pueden conducir a la modificación de conductas médicas que mejoren el pronóstico de los pacientes siempre que exista rápida disponibilidad de servicios de cardiología infan- til de calidad. $\mathrm{La} \mathrm{SpO}_{2}$ tiene buena reproducibilidad en las maternidades, recomendándose realizarla durante al menos 360 segundos ${ }^{4}$. No genera molestias a los pacientes, sólo aumentaría el tiempo de dedicación de enfermería en menos de diez minutos y ocasionaría sólo ${ }^{2,3}$ ecocardiografías normales extra por cada caso verdadero positivo.

La combinación de $\mathrm{SpO}_{2}$ positiva con hallazgos en el EF eleva la sensibilidad a $83 \%$ en niños asintomáticos al momento del alta, si bien el alto número de falsos positivos en el examen físico disminuye el VPP a 2,9.

\section{Conclusión del comentador}

El cierre del ductus, se produce gradualmente luego del nacimiento y un porcentaje de niños con cardiopatías es dado de alta sin diagnóstico, debiendo ser admitido a los pocos días en condición crítica. La combinación de un adecuado control prenatal, un EF detallado y $\mathrm{SpO} 2$ disminuyen dicho riesgo.

Gonzalo Mariani [ Sub Jefe Senicio de Neonatología. Hospital Italiano de Buenos Aires. gonzalo.mariani@ hospitalitaliano.org.ar ]

Recibido el 10/6/2009 y aceptado el 28/07/2009

Mariani G. La oximetría de pulso ayuda a detectar cardiopatías congénitas dependientes del ductus. Evid. actual. práct. Ambul; 12(4) 131, Oct-Dic 2009. Comentado de Anne de-Wahl Granelli et al Impact of pulse oximetry screening on the detection of duct dependent congenital heart disease: a Swedish prospective screening study in 39821 newborns. BMJ 2009;338;a3037. PMID 19131383.

\section{Referencias}

1. Kuehl K y col. Failure to diagnose congenital heart disease in infancy. Pediatrics 1999; 103: 743-7.

2. De-Wahl Granelli A y col. Screening for duct-dependent congenital heart disease with pulse oximetry: a critical evaluation of strategies to maximize sensitivity. Acta Paediatr 2. De-Wahl Grancll

3. Greenhalgh T. How to read a paper: Papers that report diagnostic or screening tests. BMJ 1997; 315: 540-3

4. Reich $\mathrm{J} y$ col. Reliability of a single pulse oximetry reading as a screening test for congenital heart disease in otherwise asymptomatic newborn infants: the importance of human factors. Pediatr Cardiol 2008; 29: 371-6. 